\title{
Knee subchondroplasty for management of subchondral bone cysts: a novel treatment method
}

\author{
Gerald Joseph Zeng ${ }^{1}$, MBBS, MRCs, Wei Sheng Foong ${ }^{1}$, MBBS, MMed, Tjiauw Tjoen Denny Lie ${ }^{1}$, MBBS, FRCS
}

\begin{abstract}
Knee subchondroplasty (SCP) is one of the most novel minimally invasive methods for treating bone marrow lesions. The literature suggests that it is safe, with few complications and good outcomes. However, no studies have documented its usage for managing large subchondral bone cysts. This article outlines a case report and details the pearls and pitfalls of SCP in treating large subchondral bone cysts. Our patient underwent arthroscopic debridement with medial femoral condyle SCP. Mild posterior extravasation of synthetic bone substitute was observed on Postoperative Day 1 , which was immediately rectified on revision arthroscopy. Gradual escalation of weight bearing and good pain relief were subsequently achieved, and the patient has remained complication-free after two years. No further extravasation were observed on repeat radiography. SCP is a feasible temporising measure that may help to delay the need for bone allograft or immediate knee arthroplasty in younger patients while retaining function and delaying loss of productivity.
\end{abstract}

Keywords: bone marrow lesion, clinical outcomes, knee arthroscopy, knee pain, subchondroplasty

\section{INTRODUCTION}

Knee subchondroplasty (SCP) is one of the most novel minimally invasive methods for treating bone marrow lesions (BMLs) and has garnered increasing interest in recent years. The main indication is usually prolonged (2-6 months) moderate to severe pain, with corresponding presence of BML. Created as a response to a desire to develop an alternative to partial or total knee replacement (TKA), (1) SCP procedures have witnessed a slow but steady increase in popularity for their use in treating BMLs over the past decade. It is important to address these BMLs early, as they have been shown to correlate strongly with cartilage loss, ${ }^{(2)}$ osteoarthritis and chronic pain. ${ }^{(3)}$

The literature suggests that SCP is a safe procedure with few

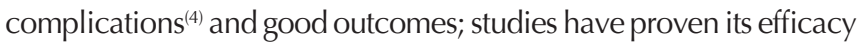
in improving function ${ }^{(5)}$ and pain. ${ }^{(6)}$ Midterm outcomes reveal high patient satisfaction rate, ${ }^{(7)}$ with more than $90 \%$ of patients expressing that they would either undergo SCP again or recommend it. Patients who failed to respond to conservative measures and were contemplating TKA showed positive results at the 2.5-year followup. Only a quarter of these patients subsequently required TKA. ${ }^{(7)}$ In those eventually requiring TKA, SCP did not compromise early outcomes or surgical performance, or increase complications. ${ }^{(8)}$

TKA is usually performed for femoral bone oedema when there are small trabecular porosities and insufficiency fractures. Despite all the discussion regarding this novel technique in treating BMLs, no studies have documented its usage for management of large subchondral bone cysts (SBCs), which should be treated owing to their strong associations with the progression of osteoarthritis. ${ }^{(9)}$ Early treatment and mitigation of injury to subchondral bone are known to prevent knee pain and halt the progression of osteoarthritis. ${ }^{(10-12)}$

The original SCP procedure involves application of a synthetic calcium phosphate-based bone substitute at the BML site to improve the structural quality of the bone in the affected region. ${ }^{(4)}$ Our study, instead, aimed to elaborate on the surgical methods and the technical challenges encountered during the SCP procedure for treatment of large cavitary SBCs. Consent was obtained from the patient for the case details and images to be published.

\section{CASE HISTORY}

On 20 June 2017, a 26-year-old woman with a significant medical history of severe aplastic anaemia was referred to our orthopaedic clinic by the haematology department. She had undergone bone marrow transplant in 2016. At presentation, she complained of chronic atraumatic right knee pain that had worsened over the past three weeks owing to exacerbation of her pre-existing medical conditions. This had resulted in difficulty in walking and limitation of function. The patient had failed to respond to prior conservative measures, including analgesics, physiotherapy and bracing.

On physical examination of the right knee, tenderness over the medial femoral condyle was elicited. The active and passive range of movement was normal, and the cruciate as well as collateral ligaments were intact. Neurovascular examination of the lower limb was normal. The limb alignment of the patient was assessed in detail before the operation to determine the need for an osteotomy in order to reduce the overload of the affected compartment. No clinical evidence of any significant varus deformity was observed. Preoperative radiographs showed symmetrical medial and lateral joint spaces (Figs. 1a \& b), consistent with our physical findings. Initial magnetic resonance (MR) imaging of the right knee (Fig. 2) revealed a focal signal BML with peripheral hyperintense rim and extensive surrounding marrow oedema in the subchondral region of the medial femoral condyle measuring $1.5 \mathrm{~cm} \times 1.9 \mathrm{~cm} \times 1.7 \mathrm{~cm}$, with

${ }^{1}$ Department of Orthopaedic Surgery, Singapore General Hospital, Singapore

Correspondence: Dr Gerald Joseph Zeng Sheng Xiang, Medical Officer, Department of Orthopaedic Surgery, Singapore General Hospital, Outram Road, Singapore 169608. geraldjosephzeng93@gmail.com 

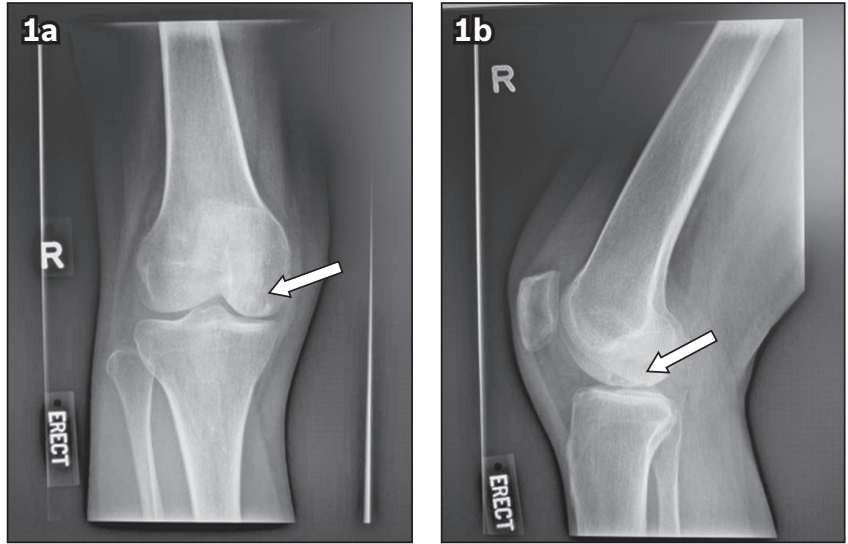

Fig. 1 Preoperative (a) anteroposterior and (b) lateral radiographs of the right knee show a radiolucent lesion in the distal medial femoral condyle, identified as a large subchondral bone cyst (arrow). Symmetrical medial and lateral joint spaces are observed.

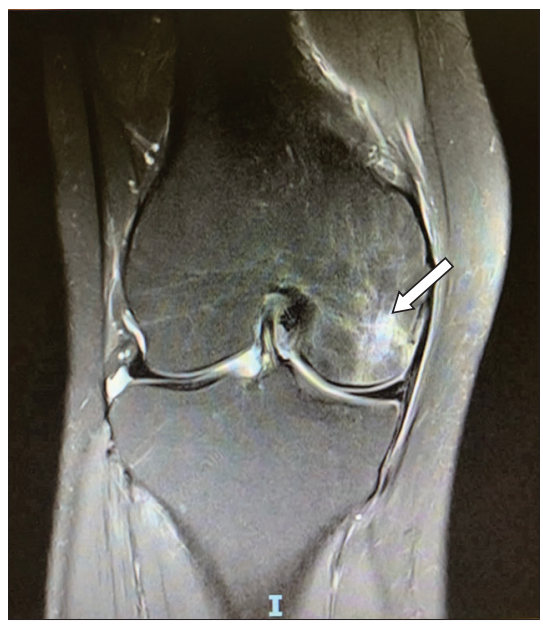

Fig. 2 Coronal MR image of the right knee shows a bone marrow lesion measuring $1.8 \mathrm{~cm} \times 2.1 \mathrm{~cm} \times 1.8 \mathrm{~cm}$, with collapse of the subchondral plate and with no evidence of a breach in the cartilage surface. The arrow indicates the area of bone marrow oedema.

no evidence of breach in the cartilage surface, which was subsequently diagnosed histopathologically via bone biopsy as chronic necrotising osteomyelitis. The patient was treated with a prolonged course of antibiotics for presumptive mycobacterial infection, then cleared by an infectious diseases specialist and conservatively treated with analgesics for six months with no improvement.

MR imaging performed seven months after medical treatment for mycobacterial infection showed interval increase in the size of the bone marrow lesion to $1.8 \mathrm{~cm} \times 2.1 \mathrm{~cm} \times 1.8 \mathrm{~cm}$, with collapse of the subchondral plate leading to an insufficiency fracture. Treatment was escalated, and the options discussed included uncompartmentalised knee arthroplasty, TKA, allograft and SCP. The potential risks and benefits of each option were thoroughly discussed. The patient eventually opted for and underwent right knee arthroscopic debridement with medial femoral condyle SCP for SBC (Figs. 3 \& 4). As the patient's gait remained preserved and no gross malalignment was noted, no additional procedures were performed apart from SCP.

\section{SURGICAL TECHNIQUE}

Examination under anaesthesia prior to arthroscopy revealed largely preserved range of motion and ligamentous stability. SCP was performed under image intensifier guidance. A navigational guide was placed over the medial femoral condyle. The FreeHand technique was utilised. FreeHand targeting used two orthogonal planes of fluoroscopic images (unobstructed anteroposterior and lateral radiographic views) to isolate the target defect. Reference was also made to MR imaging cuts examined during preoperative planning, in which we scrolled across sagittal MR image slices from the area of the lesion to the intercondylar notch to determine the cyst location relative to Blumensaat's line. Arthroscopy was previously performed for the initial survey. A fenestrated cannula was placed along a trajectory predetermined by preoperative MR imaging (studied extensively in the coronal, sagittal and axial planes) into the BML. Placement was confirmed via fluoroscopy. AccuFill ${ }^{\circledR}$ Bone Substitute Material (BSM; Zimmer Biomet, Warsaw, IN, USA) was hydrated and combined with normal saline prior to injection. Mixing was aided by the AccuMix ${ }^{\circledR}$ mixing system (Zimmer Biomet), a closed syringe device. Working time (maximum time between mixing and injecting) for the BSM was less than 15 minutes. $5 \mathrm{~mL}$ of synthetic bone substitute was injected with firm steady pressure to fill up the SBC until a darkened outline, in keeping with the pattern on the MR image, was visualised. The calcium carbonate cement was given 15 minutes to harden before commencement of arthroscopy. The AccuPort injection cannula was left in situ for at least ten minutes after cement injection to allow it to settle and to reduce the risk of extravasation. Care was taken to prevent applying excessive bending force on the cannula to prevent damage to the surrounding bone.

Subsequently, arthroscopy was performed with the patient in a supine position on a radiolucent operating table after general anaesthesia and application of a sterile thigh tourniquet. Standard arthroscopic ports were inserted to visualise and inspect the medial, lateral and patellofemoral compartments for evidence of cartilage damage. Intraoperative findings of extensive synovitis and a well-contained medial femoral condyle defect with intact cartilage were noted. Both menisci were intact. The knee was thoroughly irrigated, and detailed inspection of intraoperative radiographs showed no evidence of extravasation into the joint space (Fig. 3). This was followed by portal closure with nylon sutures.

Strict non-weight-bearing was adhered to after the operation. However, radiography on Postoperative Day (POD) 1 revealed posterior extravasation of the synthetic bone substitute (Fig. 5), which was further confirmed on revision arthroscopy (Fig. 6). This was promptly rectified during a revision procedure (Figs. 7a \& b) performed two days after the index surgery. A posteromedial portal was created to access the posterior medial aspect of the knee and remove all loose bone cement. The medial femoral condyle wall defect remained contained. Repeat plain radiography on POD 1 showed resolution, as observed in Fig. 8. 

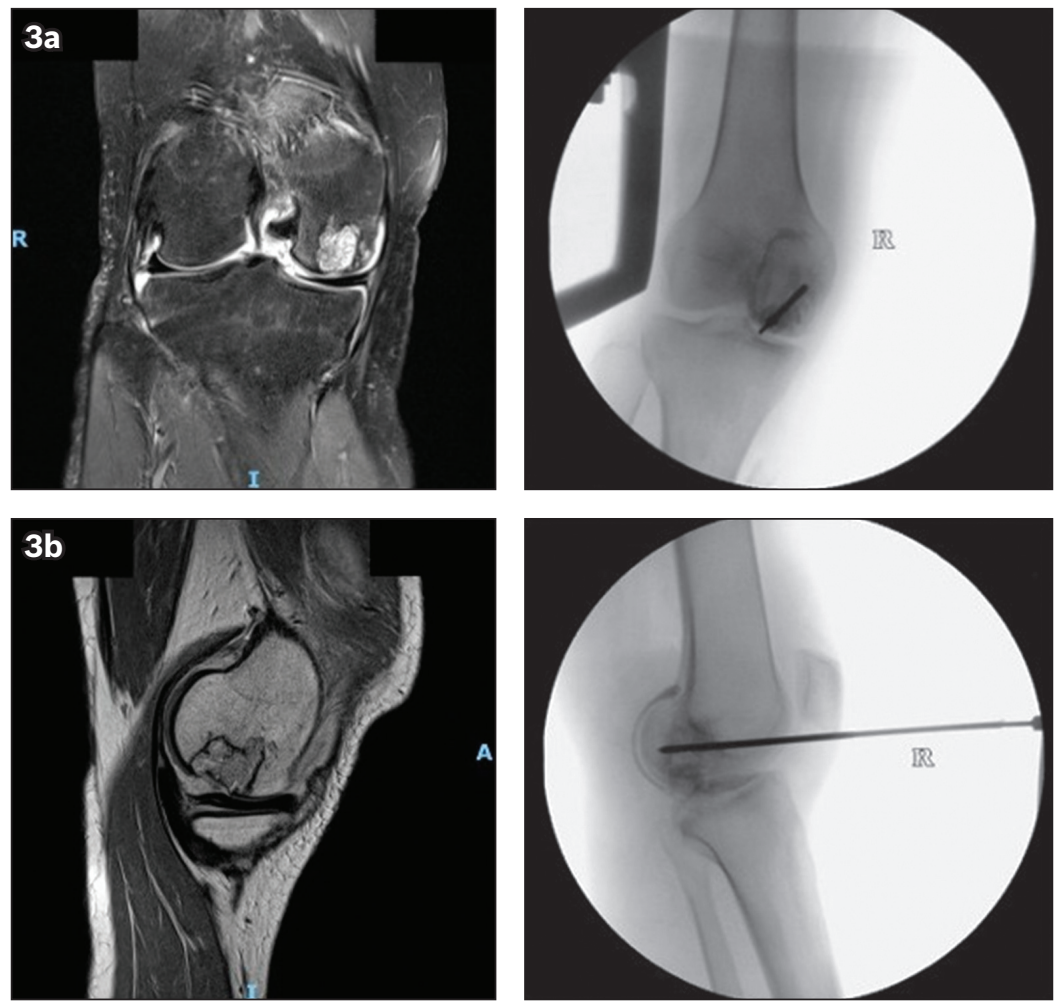

Fig. 3 Fluoroscopy images with corresponding magnetic resonance images in (a) coronal and (b) sagittal view show the subchondroplasty procedure involving injection of AccuFill ${ }^{\circledR}$ Bone Substitute Material via the FreeHand technique.

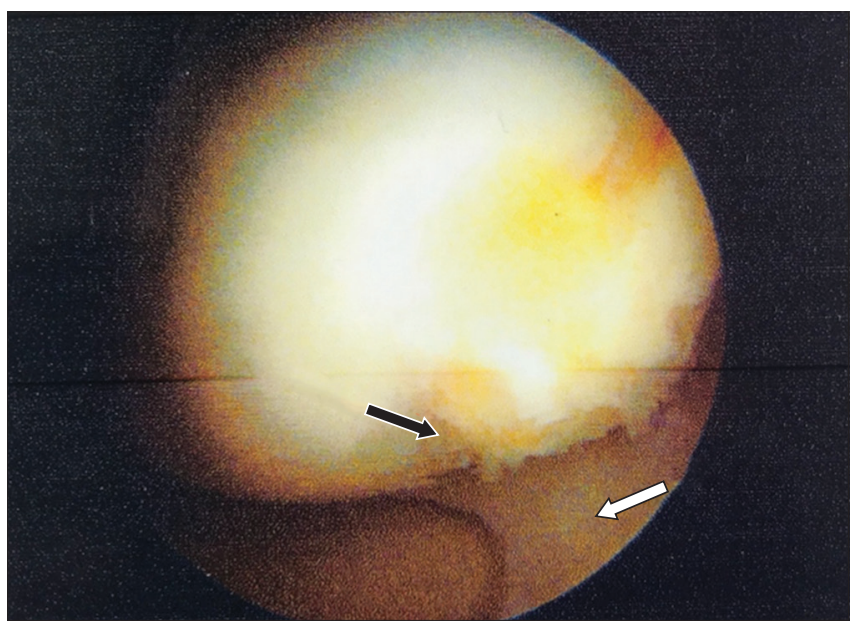

Fig. 4 Arthroscopic image of the right knee during the initial operation, after debridement with medial femoral condyle subchondroplasty, shows Outerbridge Grade 1 cartilage wear (black arrow) and an intact medial meniscus (white arrow).

Postoperative radiographs at one and two years (Fig. 9) showed gradual incorporation of the bone substitute graft, with no further extravasation noted. No progression of medial joint line narrowing was observed.

\section{RESULTS}

Gradual escalation of weight bearing was achieved over two weeks, and physical therapy was instituted immediately. Early pain relief was achieved with strict physiotherapy and adequate analgesia. At the most recent follow-up conducted two years after surgery, the patient reported improvement in symptoms, and a seven-point improvement in pain was noted on a ten-point visual

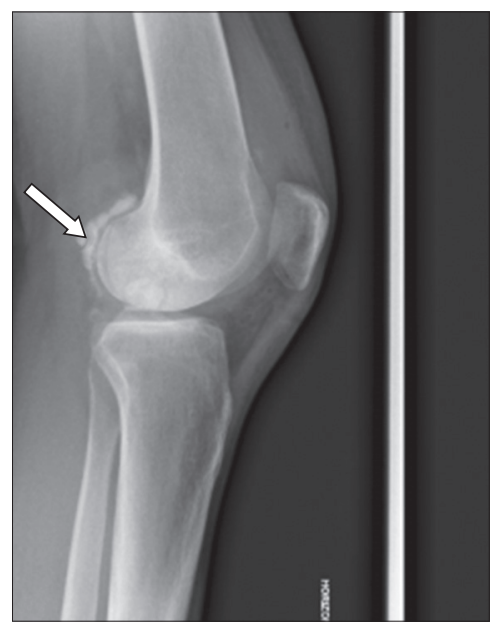

Fig. 5 Postoperative Day 1 radiograph shows posterior extravasation of bone substitute material (arrow)

analogue scale (pre-SCP 7, post-SCP 0).

No further extravasation was observed on postoperative plain radiography at two years, and the patient is planned to undergo further follow-up.

\section{DISCUSSION}

\section{Case overview}

To the best of our knowledge, this is the first documented procedure that describes successful treatment of SBC with SCP. Despite the complication of extravasation, the cyst was adequately filled up, achieving our aim of preventing collapse and providing pain relief. The success of this procedure has allowed our team to 
buy more time and reserve other surgical measures such as bone allograft or immediate knee arthroplasty for a later date. Premature employment of bone allograft is not ideal owing to its high cost. Progression to TKA would likely necessitate revision and cause unnecessary morbidity, considering the patient's young age. Our choice of patient was in keeping with the recommendations from existing literature. SCP has most frequently been performed to treat bone marrow oedema in the medial compartment (i.e. medial tibia plateau, medial femoral condyle or both). ${ }^{(13)}$ The area of interest for our procedure was the medial femoral condyle. Other possible target sites include the patella, trochlea and lateral compartment. Although our patient did not exhibit any gross contraindications, ${ }^{(4)}$ such as having a secondary cause of knee pain, ligamentous compromise or significant angular deformities, unexpected delayed extravasation occurred.

\section{Complications}

Extravasation as an immediate complication of SCP has rarely been reported. ${ }^{(14)}$ This might be partly attributable to the uncommonness and novelty of the procedure. In our case, the large size of the SBC was a predisposing factor for extravasation.

On retrospective analysis, a key consideration that might increase the risk of extravasation is the disproportionality between the volume of substrate injected and the extent of the defect. Pioneers of the technique recommend $5-16 \mathrm{~mL}$ of calcium phosphate. ${ }^{(4)}$ Strict adherence to this quantity may lead to extravasation if the volume of the defect to be filled is too small. A proposed solution to this would be to radiologically estimate the volume of the defect through preoperative imaging (e.g. computed tomography, MR imaging). Extravasation risk is further increased with (a) the presence of insufficiency fractures, which were present in our patient, or (b) multiple attempts at cannula positioning and (c) excessive pressurisation leading to overfilling. ${ }^{(15)}$ The location of the defect may also increase a predisposition to extravasation that may not be immediately noticeable. A recent article by Rebolledo et al ${ }^{(15)}$ suggested that the risk of intra-articular extravasation is more likely in femoral BMLs owing to the intracapsular area that must be traversed to access the distal femoral condyle with the cannula. Comparatively, the proximal tibia capsular reflections are more adherent, leaving less potential space for leakage into the joint. In our case, the posterior extravasation of calcium phosphate was likely precipitated by prolonged lying in the dependent position against a background of insufficiency fracture in the subchondral layer.

\section{Technical considerations}

Previous studies ${ }^{(15)}$ have recommended specific technical considerations that may improve the success of SCP, as follows: (a) a second cortical puncture, which might cause cement extravasation, should be avoided and (b) sufficient time should be allowed for the calcium phosphate cement to harden, with a recommended time of about ten minutes.

During the revision procedure, we also noted that the leaked cement particles were brittle and prone to fragmentation when an arthroscopic grasper was employed. Also, usage of suction mounted on a large-diameter arthroscopic shaver appeared to be more efficient in clearing cement debris. It may be prudent for surgeons who encounter similar situations in future to take note of this. It is also noteworthy that even in cases of extravasation, not all patients require revision arthroscopy, as some studies have shown that patients remain pain-free with conservative management. ${ }^{(14)}$ The success of an SCP procedure may also vary

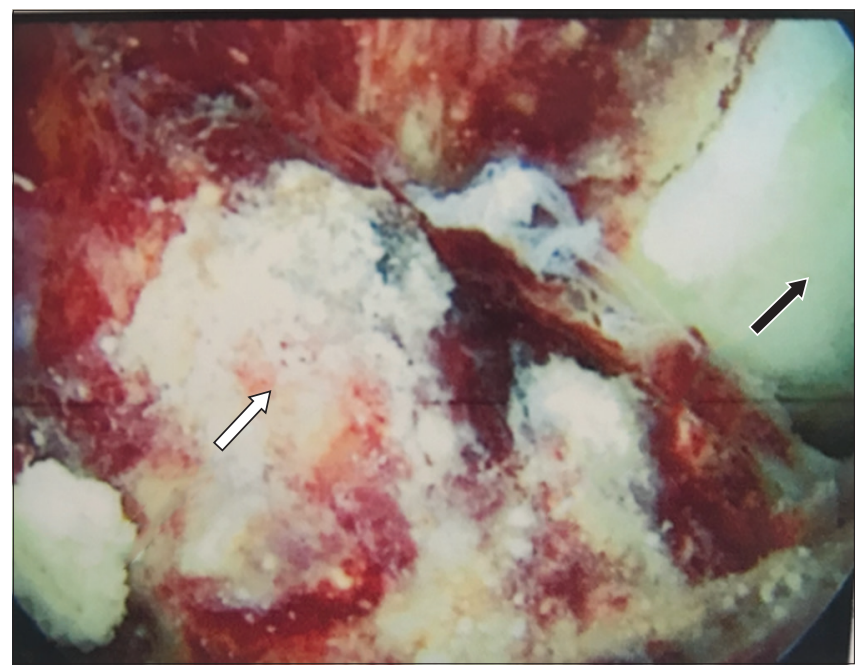

Fig. 6 Photograph of initial view on revision arthroscopy shows extravasated bone substitute material (white arrow) and the posterolateral femoral condyle (black arrow).
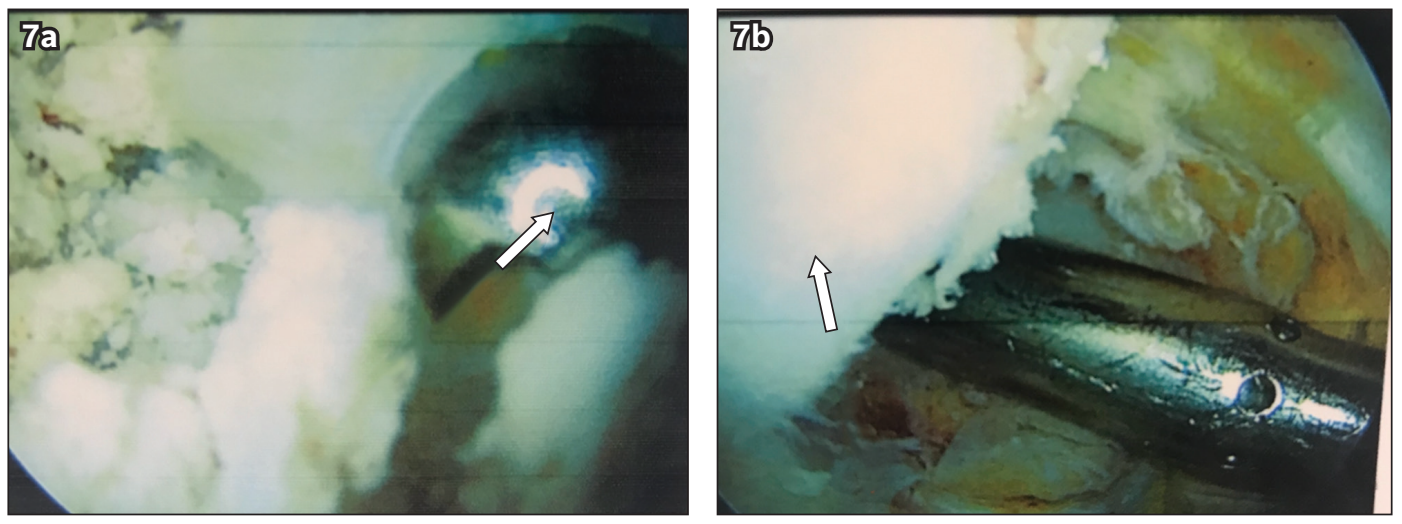

Fig. 7 (a) Photograph of revision arthroscopy shows an arthroscopic shaver (arrow) being introduced via the posterolateral portal for debridement of extravasated bone cement. (b) Photograph of the final view on revision arthroscopy after clearance of extravasated bone substitute shows the medial femoral condyle (arrow). 


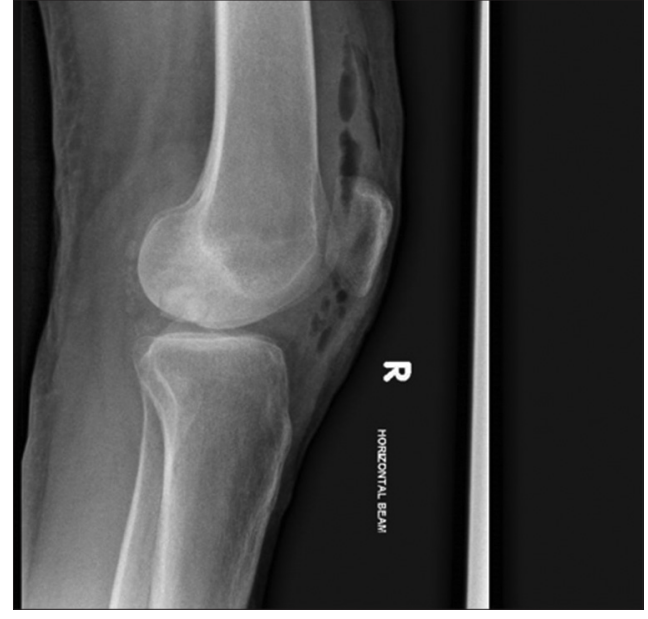

Fig. 8 Repeat plain radiograph of the right knee in lateral view, on Post-revision Operation Day 1, shows radiographic resolution of initial extravasation.

depending on the type of commercially available BSMs used. A fairly recent biomechanical study ${ }^{(16)}$ evaluating the in vitro injectability of several common commercially available BSMs demonstrated how some had failed to be successfully injected into a simulated trabecular model.

\section{Recommendations}

We recommend the use of plain radiography after SCP for surveillance of this complication. When extravasation of bone substitute cement from SCP is diagnosed, arthroscopic debridement of the extravasated material can be safely and adequately removed using arthroscopic methods.

In conclusion, SCP appears to be a safe and effective jointpreserving treatment for SBC, and may serve as a useful interim measure for younger and active patients who wish to reduce pain, avoid early arthroplasty, retain function ${ }^{(17)}$ or delay loss of productivity that may arise from major surgery. ${ }^{(1)}$ Even with cartilage restoration, recovery could take up to 12 months, and the results could be unpredictable. SCP can be a simple procedure that serves as a temporising measure. However, postoperative extravasation of material is a possible complication with potentially delayed presentation that should be promptly identified and rectified so as to facilitate recovery without permanent sequelae. Further large-scale studies with longerterm follow-up are required to further validate the true efficacy of this procedure.

\section{REFERENCES}

1. Sharkey PF, Cohen SB, Leinberry CF, Parvizi J. Subchondral bone marrow lesions associated with knee osteoarthritis. Am J Orthop (Belle Mead NJ) 2012; 41:413-7. 2. Wluka AE, Wang Y, Davies-Tuck M, et al. Bone marrow lesions predict progression
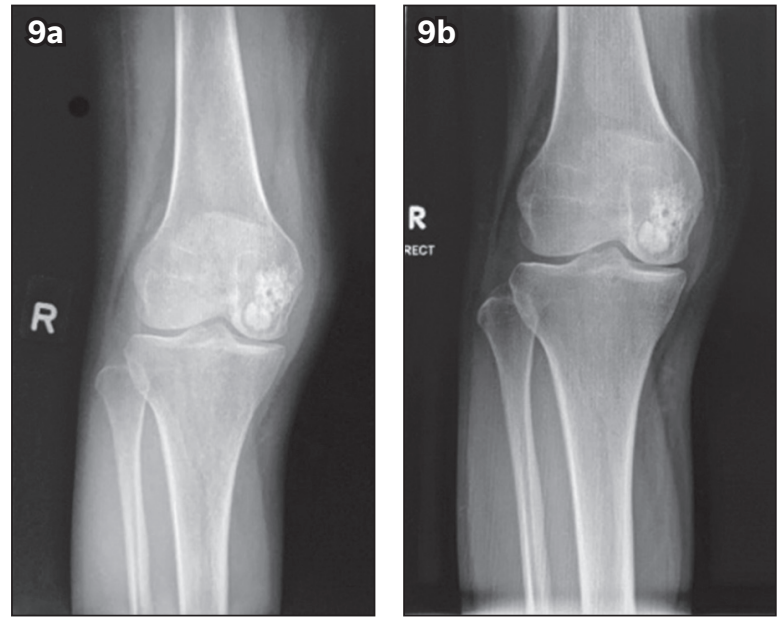

Fig. 9 Postoperative radiographs show (a) visible lucency on the periphery of the cement at one year and (b) less visible lucency surrounding the cement at two years after surgery, indicating bony growth. No further extravasation is noted, with no progression of medial joint line narrowing observed.

of cartilage defects and loss of cartilage volume in healthy middle-aged adults without knee pain over 2 yrs. Rheumatology (Oxford) 2008; 47:1392-6.

3. Felson DT, Chaisson $\mathrm{CE}$, Hill CL, et al. The association of bone marrow lesions with pain in knee osteoarthritis. Ann Intern Med 2001; 134:541-9.

4. Astur DC, de Freitas EV, Cabral PB, et al. Evaluation and management of subchondral calcium phosphate injection technique to treat bone marrow lesion. Cartilage 2019; 10:395-401.

5. Cohen SB, Sharkey PF. Subchondroplasty for treating bone marrow lesions. J Knee Surg 2016; 29:555-63.

6. Davis AT, Byrd JM, Zenner JA, et al. Short-term outcomes of the subchondroplasty procedure for the treatment of bone marrow edema lesions in patients with knee osteoarthritis. Orthop J Sports Med 2015; 3(7 Suppl 2):2325967115S00125.

7. Byrd JM, Akhavan S, Frank DA. Mid-term outcomes of the subchondroplasty procedure for patients with osteoarthritis and bone marrow edema. Orthop J Sports Med 2017; 5(7 Suppl 6):2325967117S00291.

8. Yoo JY, O'Malley MJ, Matsen Ko LJ, Cohen SB, Sharkey PF. Knee arthroplasty after subchondroplasty: early results, complications, and technical challenges. J Arthroplasty 2016; 31:2188-92.

9. Li G, Yin J, Gao J, et al. Subchondral bone in osteoarthritis: insight into risk factors and microstructural changes. Arthritis Res Ther 2013; 15:223.

10. Chatterjee D, McGee A, Strauss E, Youm T, Jazrawi L. Subchondral calcium phosphate is ineffective for bone marrow edema lesions in adults with advanced osteoarthritis. Clin Orthop Relat Res 2015; 473:2334-42.

11. Roemer FW, Neogi T, Nevitt MC, et al. Subchondral bone marrow lesions are highly associated with, and predict subchondral bone attrition longitudinally: the MOST study. Osteoarthritis Cartilage 2010; 18:47-53.

12. Tanamas SK, Wluka AE, Pelletier JP, et al. Bone marrow lesions in people with knee osteoarthritis predict progression of disease and joint replacement: a longitudinal study. Rheumatology (Oxford) 2010; 49:2413-9.

13. Farr J II, Cohen SB. Expanding applications of the subchondroplasty procedure for the treatment of bone marrow lesions observed on magnetic resonance imaging. Oper Tech Sports Med 2013; 21:138-43.

14. Bonadio MB, Giglio PN, Helito CP, et al. Subchondroplasty for treating bone marrow lesions in the knee - initial experience. Rev Bras Ortop 2017; 52:325-30.

15. Rebolledo BJ, Smith KM, Dragoo JL. Hitting the mark: optimizing the use of calcium phosphate injections for the treatment of bone marrow lesions of the proximal tibia and distal femur. Arthrosc Tech 2018; 7:e1013-8.

16. Colon DA, Yoon BJV, Russell TA, Cammisa FP, Abjornson C. Assessment of the injection behavior of commercially available bone BSMs for Subchondroplasty $®$ procedures. Knee 2015; 22:597-603.

17. Chua K, Kang JYB, Ng FDJ, et al. Subchondroplasty for bone marrow lesions in the arthritic knee results in pain relief and improvement in function. J Knee Surg 2021; 34:665-71. 